\title{
The Generalized Incompressible Navier-Stokes Equations in Besov Spaces
}

\author{
Jiahong $\mathrm{Wu}$ \\ Communicated by Charles Li, received July 21, 2004.
}

\begin{abstract}
This paper is concerned with global solutions of the generalized Navier-Stokes equations. The generalized Navier-Stokes equations here refer to the equations obtained by replacing the Laplacian in the Navier-Stokes equations by the more general operator $(-\Delta)^{\alpha}$ with $\alpha>0$. It has previously been shown that any classical solution of the $d$-dimensional generalized NavierStokes equations with $\alpha \geq \frac{1}{2}+\frac{d}{4}$ is always global in time. Thus, attention here is solely focused on the case when $\alpha<\frac{1}{2}+\frac{d}{4}$. We consider solutions emanating from initial data in several Besov spaces and establish the global existence and uniqueness of the solutions when the corresponding initial data are comparable to the diffusion coefficient in these Besov spaces.
\end{abstract}

\section{Contents}

1. Introduction

2. Besov spaces

3. $B_{p, \infty}^{r}$ solutions

4. $\dot{B}_{2,1}^{r}$ and $B_{2, q}^{r}$ Solutions 393

5. Scaling invariance 396

References

\section{Introduction}

Whether or not every smooth solution of the 3D Navier-Stokes equations is global in time has been intensively investigated but yet remains open. In this paper, we consider a more general form of the incompressible Navier-Stokes equations, namely

$$
\partial_{t} u+u \cdot \nabla u+\nabla P=-\nu(-\Delta)^{\alpha} u, \quad \nabla \cdot u=0,
$$

1991 Mathematics Subject Classification. Primary 35Q30; Secondary 76D03.

Key words and phrases. The generalized Navier-Stokes equations, global solutions, Besov spaces. 
where $\nu>0$ and $\alpha>0$ are real parameters. (1.1) becomes the Navier-Stokes equations in the case of $\alpha=1$ and will thus be referred to as the generalized Navier-Stokes (GNS) equations. The goal of this paper is to establish the global existence and uniqueness of solutions of (1.1) when the corresponding initial data

$$
u(x, 0)=u_{0}(x)
$$

are prescribed in several functional spaces detailed below.

We consider the general $d$-dimensional GNS equations. When $\alpha \geq \frac{1}{2}+\frac{d}{4}$, any classical solution of (1.1) is always global in time $([\mathbf{1 4}])$. In particular, smooth solutions of the 2D Navier-Stokes equations and the 3D GNS equations with $\alpha \geq \frac{5}{4}$ do not develop finite-time singularities.

This paper focuses its attention on the case when $\alpha<\frac{1}{2}+\frac{d}{4}$. We seek global solutions emanating from initial data in several Besov spaces. Besov spaces include many of the frequently-used function spaces such as the Sobolev spaces and the Hölder spaces and constitute a very natural setting for studying solutions of various partial differential equations $([\mathbf{1}],[\mathbf{4}],[\mathbf{5}],[\mathbf{7}],[\mathbf{1 2}],[\mathbf{1 3}],[\mathbf{1 5}])$. Our study here covers the inhomogeneous Besov spaces $B_{p, \infty}^{r}$ and $B_{2, q}^{r}$, and the homogeneous Besov space $\dot{B}_{2,1}^{r}$. Our major results can be roughly summarized as follows. Assuming $\nu>0$ and $\alpha<\frac{1}{2}+\frac{d}{4}$, the GNS equations (1.1) have a unique and global solution when the norm of $u_{0}$ is comparable to $\nu$ in any one of the spaces:

i) $B_{p, \infty}^{r}$ with $1 \leq p \leq 2, r>1$ and $r>1+\frac{d}{p}-2 \alpha$;

ii) $B_{2,1}^{r}$ with $r>1$ and $r \geq 1+\frac{d}{2}-2 \alpha$;

iii) $\dot{B}_{2,1}^{r}$ with $r=1+\frac{d}{2}-2 \alpha>1$;

iv) $B_{2, q}^{r}$ with $1<q<\infty, r>1$ and $r>1+\frac{d}{2}-\frac{2 \alpha}{q}$.

Precise statements and their proofs will be deferred until Section 3 and Section 4. A particular consequence of these results is the global existence of solutions starting with data in the usual Sobolev space $H^{r}$ with $r>1+\frac{d}{2}-2 \alpha$.

The index $r=1+\frac{d}{2}-2 \alpha$ appears to be critical in a sense that we now explain. Solutions of the GNS equations (1.1) are scaling invariant. That is, if $(u, P)$ is a solution of the GNS equations, then $\left(u_{\lambda}, P_{\lambda}\right)$ is also a solution of the GNS equations, where

$$
u_{\lambda}(x, t)=\lambda^{2 \alpha-1} u\left(\lambda x, \lambda^{2 \alpha} t\right), \quad P_{\lambda}(x, t)=\lambda^{4 \alpha-2} P\left(\lambda x, \lambda^{2 \alpha} t\right) .
$$

As we shall show in Section 5 , the norm of $u_{\lambda}$ is virtually invariant in the homogeneous Besov spaces $\dot{B}_{2, \infty}^{1+\frac{d}{2}-2 \alpha}$ and $\dot{B}_{2,1}^{1+\frac{d}{2}-2 \alpha}$, namely

$$
\left\|u_{\lambda}\right\|_{\dot{B}_{2, \infty}^{1+\frac{d}{2}-2 \alpha}} \approx\|u\|_{\dot{B}_{2, \infty}^{1+\frac{d}{2}-2 \alpha}}, \quad\left\|u_{\lambda}\right\|_{\dot{B}_{2,1}^{1+\frac{d}{2}-2 \alpha}} \approx\|u\|_{\dot{B}_{2,1}^{1+\frac{d}{2}-2 \alpha}}
$$

where $\approx$ means the equivalence between two norms. These invariance properties allow one to argue that the index restriction in iii) may not be relaxed to $r \geq$ $1+\frac{d}{2}-2 \alpha$ and that the inhomogeneous Besov space in i) with $p=2$ may not be replaced by the homogeneous Besov space $\dot{B}_{2, \infty}^{r}$ with $r>1$ and $r>1+\frac{d}{2}-2 \alpha$. Further explanations will be provided in Section 5 .

We remark that Cannone, Planchon, Lemarié-Rieusset and others have previously studied mild solutions of the Navier-Stokes equations in Besov spaces via the fixed point arguments based on the continuity of the bilinear form in these spaces. 
One related result is the small-data global (in time) existence of mild solutions in the homogeneous Besov spaces $\dot{B}_{p, \infty}^{\frac{3}{p}-1}$ with $1<p<3([\mathbf{3}])$. This result was later extended to $\dot{B}_{p, \infty}^{\frac{3}{p}-1}$ with $p>3$, but the uniqueness of such mild solutions is unknown $([\boldsymbol{8}])$. In comparison, this paper is mainly concerned with solutions in more regular Besov spaces in which the solutions of the GNS equations can be shown to be unique.

Before presenting our major results in Section 3 and Section 4, we provide the definitions of Besov spaces and some embedding relations and inequalities in Section 2. We also need several other inequalities involving Besov spaces, which are left to the Appendix.

\section{Besov spaces}

In this section, we provide the definitions of the homogeneous and the inhomogeneous Besov spaces. They are defined through the Littlewood-Paley decomposition. Several related embedding relations and inequalities will also be given here. Except for Proposition 2.3, most of the materials in this section are classical and we refer the reader to the books $[\mathbf{2}],[\mathbf{6}],[\mathbf{9}],[\mathbf{1 0}]$ for more details.

We start with the Fourier transform. The Fourier transform $\widehat{f}$ of a $L^{1}$-function $f$ is given by

$$
\widehat{f}(\xi)=\int_{\mathbb{R}^{d}} f(x) e^{-2 \pi x \cdot \xi} d x .
$$

More generally, the Fourier transform of any $f \in \mathcal{S}^{\prime}$, the space of tempered distributions, is given by

$$
(\widehat{f}, g)=(f, \widehat{g})
$$

for any $g \in \mathcal{S}$, the usual Schwarz class. The Fourier transform is a bounded linear bijection from $\mathcal{S}^{\prime}$ to $\mathcal{S}^{\prime}$ whose inverse is also bounded.

The fractional power of the Laplacian can be defined in terms of the Fourier transform. For a general exponent $\beta \in \mathbb{R}$,

$$
\left(-\widehat{\Delta)^{\beta / 2}} f(\xi)=(2 \pi|\xi|)^{\beta} \widehat{f}(\xi) .\right.
$$

For notational convenience, we will write $\Lambda$ for $(-\Delta)^{1 / 2}$ from now on. Another important family of operators are the Riesz transforms. For $1 \leq l \leq d$,

$$
\widehat{\mathcal{R}_{l} f}(\xi)=-i \frac{\xi_{l}}{|\xi|} \widehat{f}(\xi)
$$

To define the Besov spaces, we fix some notation.

$$
\mathcal{S}_{0}=\left\{\phi \in \mathcal{S}, \int_{\mathbb{R}^{d}} \phi(x) x^{\gamma} d x=0,|\gamma|=0,1,2, \cdots\right\} .
$$

Its dual is given by

$$
\mathcal{S}_{0}^{\prime}=\mathcal{S}^{\prime} / \mathcal{S}_{0}^{\perp}=\mathcal{S}^{\prime} / \mathcal{P},
$$

where $\mathcal{P}$ is the space of multinomials. In other words, two distributions in $\mathcal{S}_{0}^{\prime}$ are identified as the same if their difference is a multinomial. 
We now introduce a dyadic partition of $\mathbb{R}^{d}$. We choose $\phi_{0} \in \mathcal{S}\left(\mathbb{R}^{d}\right)$ such that $\phi_{0}$ is even,

$$
\operatorname{supp} \phi_{0}=\left\{\xi: 2^{-1} \leq|\xi| \leq 2\right\}, \quad \text { and } \phi_{0}>0 \text { on } A_{0},
$$

where $A_{j}=\left\{\xi: 2^{j-1}<|\xi|<2^{j+1}\right\}$ for $j \in \mathbb{Z}$. For $j \in \mathbb{Z}$, define

$$
\phi_{j}(\xi)=\phi_{0}\left(2^{-j} \xi\right)
$$

and define $\Phi_{j} \in \mathcal{S}$ by

$$
\widehat{\Phi}_{j}(\xi)=\frac{\phi_{j}(\xi)}{\sum_{j} \phi_{j}(\xi)} .
$$

It follows that both $\widehat{\Phi}_{j}$ and $\Phi_{j}$ are even and satisfy the following properties:

$$
\widehat{\Phi}_{j}(\xi)=\widehat{\Phi}_{0}\left(2^{-j} \xi\right), \quad \operatorname{supp} \widehat{\Phi}_{j} \subset A_{j}, \quad \Phi_{j}(x)=2^{j d} \Phi_{0}\left(2^{j} x\right) .
$$

Furthermore,

$$
\sum_{k=-\infty}^{\infty} \widehat{\Phi}_{k}(\xi)= \begin{cases}1, & \text { if } \xi \in \mathbb{R}^{d} \backslash\{0\}, \\ 0, & \text { if } \xi=0 .\end{cases}
$$

Thus, for a general function $\psi \in \mathcal{S}$, we have

$$
\sum_{k=-\infty}^{\infty} \widehat{\Phi}_{k}(\xi) \widehat{\psi}(\xi)=\widehat{\psi}(\xi) \quad \text { for } \xi \in \mathbb{R}^{d} \backslash\{0\} .
$$

But, if $\psi \in \mathcal{S}_{0}$, then

$$
\sum_{k=-\infty}^{\infty} \widehat{\Phi}_{k}(\xi) \widehat{\psi}(\xi)=\widehat{\psi}(\xi) \quad \text { for all } \xi \in \mathbb{R} .
$$

That is, for $\psi \in \mathcal{S}_{0}$,

and hence

$$
\sum_{k=-\infty}^{\infty} \Phi_{k} * \psi=\psi
$$

$$
\sum_{k=-\infty}^{\infty} \Phi_{k} * f=f
$$

in the weak* topology of $\mathcal{S}_{0}^{\prime}$ for any $f \in \mathcal{S}_{0}^{\prime}$.

Now let $\Psi \in C_{0}^{\infty}\left(\mathbb{R}^{d}\right)$ be even and satisfy

$$
\widehat{\Psi}(\xi)=1-\sum_{k=0}^{\infty} \widehat{\Phi}_{k}(\xi) .
$$

Then, for any $\psi \in \mathcal{S}$,

$$
\Psi * \psi+\sum_{0}^{\infty} \Phi_{k} * \psi=\psi
$$

and hence

$$
\Psi * f+\sum_{k=0}^{\infty} \Phi_{k} * f=f
$$

in $\mathcal{S}^{\prime}$ for any $f \in \mathcal{S}^{\prime}$.

To define the homogeneous Besov spaces, we set

$$
\Delta_{j} f=\Phi_{j} * f, \quad j=0, \pm 1, \pm 2, \cdots .
$$


Suppose that $s \in \mathbb{R}$ and $p, q \in[1, \infty]$. We say that $f \in \dot{B}_{p, q}^{s}$ if $f \in \mathcal{S}_{0}^{\prime}$ and

$$
\sum_{j=-\infty}^{\infty}\left(2^{j s}\left\|\Delta_{j} f\right\|_{L^{p}}\right)^{q}<\infty
$$

$\dot{B}_{p, q}^{s}$ is a Banach space when equipped with the norm

$$
\|f\|_{\dot{B}_{p, q}^{s}} \equiv \begin{cases}\left(\sum_{j=-\infty}^{\infty}\left(2^{j s}\left\|\Delta_{j} f\right\|_{L^{p}}\right)^{q}\right)^{1 / q}, & \text { if } q<\infty, \\ \sup _{-\infty<j<\infty} 2^{j s}\left\|\Delta_{j} f\right\|_{L^{p}}, & \text { if } q=\infty .\end{cases}
$$

$\dot{B}_{p, q}^{s}$ with this norm will be referred to as homogeneous Besov space.

To introduce the inhomogeneous Besov spaces, we define

$$
\Delta_{j} f= \begin{cases}0, & \text { if } j \leq-2, \\ \Psi * f, & \text { if } j=-1, \\ \Phi_{j} * f, & \text { if } j=0,1,2, \cdots\end{cases}
$$

For $s \in \mathbb{R}$ and $p, q \in[1, \infty]$, we say that $f \in B_{p, q}^{s}$ if $f \in \mathcal{S}^{\prime}$ and

$$
\left\|\Delta_{-1} f\right\|_{L^{p}}+\left(\sum_{j=0}^{\infty}\left(2^{j s}\left\|\Delta_{j} f\right\|_{L^{p}}\right)^{q}\right)^{1 / q}<\infty .
$$

$B_{p, q}^{s}$ is a Banach space with the norm

$$
\|f\|_{B_{p, q}^{s}} \equiv \begin{cases}\left\|\Delta_{-1} f\right\|_{L^{p}}+\left(\sum_{j=0}^{\infty}\left(2^{j s}\left\|\Delta_{j} f\right\|_{L^{p}}\right)^{q}\right)^{1 / q}, & \text { if } q<\infty, \\ \left\|\Delta_{-1} f\right\|_{L^{p}}+\sup _{0 \leq j<\infty} 2^{j s}\left\|\Delta_{j} f\right\|_{L^{p}}, & \text { if } q=\infty .\end{cases}
$$

$B_{p, q}^{s}$ with this norm will be referred to as inhomogeneous Besov space.

We now point out several simple facts concerning the operators $\Delta_{j}$ :

$$
\begin{aligned}
& \Delta_{j} \Delta_{k}=0, \text { if }|j-k| \geq 2 ; \\
& S_{j} \equiv \sum_{k=-\infty}^{j} \Delta_{k} \rightarrow I, \text { as } j \rightarrow \infty ; \\
& \Delta_{k}\left(S_{j-1} f \Delta_{j} f\right)=0, \text { if }|j-k| \geq 4 .
\end{aligned}
$$

$I$ in (2.9) denotes the identity operator and (2.9) is simply another way of writing (2.2) and (2.3). Finally, we caution that $\Delta_{j}$ with $j \leq-1$ associated with the homogeneous Besov space $\dot{B}_{p, q}^{s}$ are defined differently from those associated with the inhomogeneous Besov space $B_{p, q}^{s}$. Therefore, it will be understood that $\Delta_{j}$ with $j \leq-1$ in the context of the homogeneous Besov space are given by (2.4) and by (2.6) in the context of the inhomogeneous Besov space.

The Besov spaces defined above obey various inclusion relations. In particular, we have the following theorem.

Theorem 2.1. Assume that $\beta \in \mathbb{R}$ and $p, q \in[1, \infty]$.

1) If $\beta>0$, then $B_{p, q}^{\beta} \subset \dot{B}_{p, q}^{\beta}$. 
2) If $\beta_{1} \leq \beta_{2}$, then $B_{p, q}^{\beta_{2}} \subset B_{p, q}^{\beta_{1}}$. This inclusion relation is false for the homogeneous Besov spaces.

3) If $1 \leq q_{1} \leq q_{2} \leq \infty$, then $\dot{B}_{p, q_{1}}^{\beta} \subset \dot{B}_{p, q_{2}}^{\beta}$ and $B_{p, q_{1}}^{\beta} \subset B_{p, q_{2}}^{\beta}$.

4) (Besov embedding theorem) If $1 \leq p_{1} \leq p_{2} \leq \infty$ and $\beta_{1}=\beta_{2}+d\left(\frac{1}{p_{1}}-\right.$ $\left.\frac{1}{p_{2}}\right)$, then $\dot{B}_{p_{1}, q}^{\beta_{1}}\left(\mathbb{R}^{d}\right) \subset \dot{B}_{p_{2}, q}^{\beta_{2}}\left(\mathbb{R}^{d}\right)$ and $B_{p_{1}, q}^{\beta_{1}}\left(\mathbb{R}^{d}\right) \subset B_{p_{2}, q}^{\beta_{2}}\left(\mathbb{R}^{d}\right)$.

In addition, the usual Sobolev spaces are a special type of Besov spaces and thus follow similar embedding relations. We recall that for $\beta \geq 0$,

$$
\dot{H}^{\beta}=\left\{f \in \mathcal{S}^{\prime}:|\xi|^{\beta}|\widehat{f}(\xi)| \in L^{2}\right\}
$$

and

It is not hard to check that

$$
H^{\beta}=\left\{f \in \mathcal{S}^{\prime}:\left(1+|\xi|^{2}\right)^{\beta / 2}|\widehat{f}(\xi)| \in L^{2}\right\} .
$$

$$
\dot{H}^{\beta}\left(\mathbb{R}^{d}\right)=\dot{B}_{2,2}^{\beta}\left(\mathbb{R}^{d}\right) \quad \text { and } \quad H^{\beta}\left(\mathbb{R}^{d}\right)=B_{2,2}^{\beta}\left(\mathbb{R}^{d}\right) .
$$

By 3) of Theorem 2.1 is

$$
\dot{B}_{2,1}^{\beta} \subset \dot{H}^{\beta} \subset \dot{B}_{2, \infty}^{\beta}, \quad B_{2,1}^{\beta} \subset H^{\beta} \subset B_{2, \infty}^{\beta} .
$$

We now turn to Bernstein's inequalities. When the Fourier transform of a function is supported on a ball or an annulus, the $L^{p}$-norms of its derivatives can be bounded in terms of the norms of the function itself. Inequalities of this nature are referred to as Bernstein's inequalities. The classical Bernstein's inequalities only allow integer derivatives. They can actually be extended to involve fractional derivatives. In the following, we shall first state as a proposition the classical Bernstein's inequalities and then present the fractional Bernstein inequalities.

Proposition 2.2. Let $k \geq 0$ be an integer and $1 \leq p \leq q \leq \infty$.

1) If supp $\widehat{f} \subset\left\{\xi \in \mathbb{R}^{d}:|\xi| \leq c \lambda\right\}$, then

$$
\sup _{|\gamma|=k}\left\|D^{\gamma} f\right\|_{L^{q}} \leq c \lambda^{k+d(1 / p-1 / q)}\|f\|_{L^{p}} .
$$

2) If supp $\widehat{f} \subset\left\{\xi \in \mathbb{R}^{d}: c_{1} \lambda \leq|\xi| \leq c_{2} \lambda\right\}$, then

$$
c_{3} \lambda^{k+d(1 / p-1 / q)} \leq \sup _{|\gamma|=k}\left\|D^{\gamma} f\right\|_{L^{q}} \leq c_{4} \lambda^{k+d(1 / p-1 / q)}\|f\|_{L^{p}},
$$

where $c, c_{1}, c_{2}, c_{3}$ and $c_{4}$ are constants independent of $\lambda$.

The proof of this proposition is classical and can be found in [6]. We now state the generalized Bernstein's inequalities involving fractional derivatives. In the following proposition, we still use $c$ (or $c$ with a subindex) to denote various constants whose values may be different from line to line. Occasionally, we use $C$ with a subindex to mark some crucial constants.

Proposition 2.3. Assume that $\beta \in \mathbb{R}$ and $1 \leq p \leq q \leq \infty$.

1) If $\beta \geq 0$ and supp $\widehat{f} \subset\left\{\xi \in \mathbb{R}^{d}:|\xi| \leq c \lambda\right\}$, then

$$
\left\|\Lambda^{\beta} f\right\|_{L^{q}} \leq c \lambda^{\beta+d(1 / p-1 / q)}\|f\|_{L^{p}},
$$

2) If supp $\widehat{f} \subset\left\{\xi \in \mathbb{R}^{d}: c_{1} \lambda \leq|\xi| \leq c_{2} \lambda\right\}$, then

$$
c_{3} \lambda^{\beta+d(1 / p-1 / q)} \leq\left\|\Lambda^{\beta} f\right\|_{L^{q}} \leq c_{4} \lambda^{\beta+d(1 / p-1 / q)}\|f\|_{L^{p}} .
$$


Proposition 2.3 is a simple extension of Proposition 2.2. The statements in Proposition 2.3 are communicated to the author by David Ullrich [11].

To establish the major results of this paper, we also need several other inequalities involving Besov spaces. They include the logarithmic Besov inequalities, two commutator estimates, and some estimates for the usual product of two functions in Besov spaces. Instead of presenting them here, we leave to the Appendix.

\section{3. $B_{p, \infty}^{r}$ solutions}

In this section, we study solutions of the initial-value problem (IVP) for the GNS equations, namely

$$
\left\{\begin{array}{l}
\partial_{t} u+u \cdot \nabla u+\nabla P=-\nu(-\Delta)^{\alpha} u \\
\nabla \cdot u=0 \\
u(x, 0)=u_{0}(x)
\end{array}\right.
$$

Attention will be mainly focused on $u_{0} \in B_{2, \infty}^{r}\left(\mathbb{R}^{d}\right)$. Our goal is to establish the existence and uniqueness of solutions to (3.1) with $u_{0} \in B_{2, \infty}^{r}$ satisfying suitable conditions. The major results are presented in Theorem 3.2. For the purpose of proving this theorem, we first present an a priori estimate stated in Proposition 3.1. We remark that Theorem 3.2 can be extended to cover any initial datum in $B_{p, \infty}^{s}$ with $1 \leq p \leq 2$ through an embedding theorem.

We start with an important a priori estimate.

Proposition 3.1. Let $r \in \mathbb{R}$ and $s>1+\frac{d}{2}$. Then any solution $(u, P)$ of the IVP (3.1) obeys the following differential inequality

$$
\frac{d}{d t}\|u\|_{B_{2, \infty}^{r}}+c \nu\|u\|_{B_{2, \infty}^{r+2 \alpha}} \leq c\|u\|_{B_{2, \infty}^{s}}\|u\|_{B_{2, \infty}^{r}},
$$

where c's are constants with possible dependence on $r$ and $s$ only.

Proposition 3.1 contains a major ingredient in proving Theorem 3.2 stated below. As we have mentioned in the introduction, the issue of global smooth solutions has been resolved for (3.1) with $\alpha>\frac{1}{2}+\frac{d}{4}$. Therefore, we shall assume that $\alpha<\frac{1}{2}+\frac{d}{4}$ here.

Theorem 3.2. Let $\nu>0$ and $\alpha<\frac{1}{2}+\frac{d}{4}$. Assume that $u_{0} \in B_{2, \infty}^{r}$ with

and satisfies

$$
r>1, \quad r>1+\frac{d}{2}-2 \alpha
$$

$$
\left\|u_{0}\right\|_{B_{2, \infty}^{r}} \leq C_{0} \nu
$$

for some suitable constant $C_{0}$. Then the IVP (3.1) has a unique global solution $(u, P)$ satisfying

$$
\begin{gathered}
u \in L^{\infty}\left([0, \infty) ; B_{2, \infty}^{r}\right) \cap L^{1}\left([0, \infty) ; B_{2, \infty}^{r+2 \alpha}\right) \cap C\left([0, \infty) ; B_{2, \infty}^{r-1}\right), \\
P \in L^{1}\left([0, \infty) ; B_{2, \infty}^{r}\right)
\end{gathered}
$$

and

$$
\|u(\cdot, t)\|_{B_{2, \infty}^{r}} \leq 2 C_{0} \nu, \quad \text { for all } t>0 .
$$


We make two remarks.

Remark. Using the Besov embedding theorem (Theorem 2.1), we can extend Theorem 3.2 to cover any initial datum $u_{0} \in B_{p, \infty}^{s}$ with $1 \leq p \leq 2$. In fact, Theorem 2.1 states that for $1 \leq p \leq 2$ and $s=r+d\left(\frac{1}{p}-\frac{1}{2}\right)$,

$$
B_{p, \infty}^{s} \subset B_{2, \infty}^{r} \text {. }
$$

Thus, $u_{0} \in B_{p, \infty}^{s}$ with $1 \leq p \leq 2$ and $s>1+\frac{d}{p}-2 \alpha$ implies that $u_{0} \in B_{2, \infty}^{r}$ with $r>1+\frac{d}{2}-2 \alpha$. Therefore, if $u_{0} \in B_{p, \infty}^{s}$ with

$$
1 \leq p \leq 2, \quad s>1, \quad s>1+\frac{d}{p}-2 \alpha,
$$

then Theorem 3.2 implies that (3.1) has a unique global solution.

Remark. Because of the embedding relations in (2.11), namely

$$
H^{r}=B_{2,2}^{r} \subset B_{2, \infty}^{r},
$$

another special consequence of Theorem 3.2 is the global existence and uniqueness of solutions of (3.1) corresponding to any initial datum in the usual Sobolev space $H^{r}$ with $r>1+\frac{d}{2}-2 \alpha$.

We now proceed to the proofs of Proposition 3.1 and Theorem 3.2.

Proof of Proposition 3.1. For each $j \in \mathbb{Z}$, we apply $\Delta_{j}$ to the GNS equations in $(3.1)$

$$
\partial_{t} \Delta_{j} u+u \cdot \nabla \Delta_{j} u+\nu(-\Delta)^{\alpha} \Delta_{j} u=\left[u \cdot \nabla, \Delta_{j}\right] u-\nabla \Delta_{j} P
$$

where the brackets $[$,$] in \left[u \cdot \nabla, \Delta_{j}\right]$ represents the commutator, namely

$$
\left[u \cdot \nabla, \Delta_{j}\right] u=u \cdot \nabla \Delta_{j} u-\Delta_{j}(u \cdot \nabla u) .
$$

Multiplying by $\Delta_{j} u$ and integrating with respect to $x$ leads to

$$
\frac{d}{d t}\left\|\Delta_{j} u\right\|_{L^{2}}^{2}+\nu I=I I+I I I
$$

where

$$
\begin{aligned}
& I=\int\left|\Lambda^{\alpha} \Delta_{j} u\right|^{2} d x, \\
& I I=\int\left[u \cdot \nabla, \Delta_{j}\right] u \cdot \Delta_{j} u d x, \\
& I I I=-\int\left(\nabla \Delta_{j} P\right) \cdot \Delta_{j} u d x .
\end{aligned}
$$

We now evaluate these terms. By Proposition 2.3, I has the following lower bound

$$
I \geq c 2^{2 \alpha j}\left\|\Delta_{j} u\right\|_{L^{2}}^{2} .
$$

To deal with $I I$, we first apply Hölder's inequality and then the commutator estimate in Proposition A.2 to obtain

$$
I I \leq\left\|\Delta_{j} u\right\|_{L^{2}}\left\|\left[u \cdot \nabla, \Delta_{j}\right] u\right\|_{L^{2}} \leq c\|\nabla u\|_{L^{\infty}}\left\|\Delta_{j} u\right\|_{L^{2}}^{2} .
$$

The estimate of $I I I$ is more complex and the following lemma is devoted to it. 
Lemma 3.3. For $j \in \mathbb{Z}$ and any solution $(u, P)$ of the IVP (3.1), we have

$$
\left|\int\left(\Delta_{j} \nabla P\right) \cdot \Delta_{j} u d x\right| \leq c\|\nabla u\|_{L^{\infty}}\left\|\Delta_{j} u\right\|_{L^{2}}^{2} .
$$

Proof of Lemma 3.3. Applying $\nabla$. to the first equation in (3.1) and using the second equation, we have

$$
P=-\mathcal{R}_{k} \mathcal{R}_{l}\left(u_{k} u_{l}\right),
$$

where $\mathcal{R}$ with a subindex denotes a $2 \mathrm{D}$ Riesz transform, and the repeated indices $k$ and $l$ are summed. Therefore,

$$
\begin{aligned}
-\Delta_{j} \nabla P= & \mathcal{R}_{k} \mathcal{R}_{l}\left(-\Delta_{j}\left(u_{l} \nabla u_{k}\right)-\Delta_{j}\left(u_{k} \nabla u_{l}\right)\right) \\
= & \mathcal{R}_{k} \mathcal{R}_{l}\left(\left[u_{l} \nabla, \Delta_{j}\right] u_{k}+\left[u_{k} \nabla, \Delta_{j}\right] u_{l}\right) \\
& \quad-\mathcal{R}_{k} \mathcal{R}_{l}\left(u_{l} \nabla\left(\Delta_{j} u_{k}\right)+u_{k} \nabla\left(\Delta_{j} u_{l}\right)\right) \\
= & I I I_{1}+I I I_{2} .
\end{aligned}
$$

Correspondingly, the integral to be bounded is divided into two parts:

$$
\int\left(\Delta_{j} \nabla P\right) \cdot \Delta_{j} u d x=\int I I I_{1} \cdot \Delta_{j} u d x+\int I I I_{2} \cdot \Delta_{j} u d x .
$$

For the first integral, we have

$$
\left|\int I I I_{1} \cdot \Delta_{j} u d x\right| \leq\left\|\Delta_{j} u\right\|_{L^{2}}\left\|I I I_{1}\right\|_{L^{2}} \leq\left\|\Delta_{j} u\right\|_{L^{2}} \sum_{j, k}\left\|\left[u_{l} \nabla, \Delta_{j}\right] u_{k}\right\|_{L^{2}}
$$

Applying the commutator estimate in Proposition A.2 yields

$$
\left|\int I I I_{1} \cdot \Delta_{j} u d x\right| \leq c\|\nabla u\|_{L^{\infty}}\left\|\Delta_{j} u\right\|_{L^{2}}^{2} .
$$

To bound the second term in (3.8), we integrate by parts,

$$
\begin{aligned}
\int I I I_{2} \cdot \Delta_{j} u d x= & -\int \mathcal{R}_{k} \mathcal{R}_{l}\left(u_{l} \partial_{m}\left(\Delta_{j} u_{k}\right)\right) \Delta_{j} u_{m} d x \\
& -\int \mathcal{R}_{k} \mathcal{R}_{l}\left(u_{k} \partial_{m}\left(\Delta_{j} u_{l}\right)\right) \Delta_{j} u_{m} d x \\
= & \int \mathcal{R}_{k} \mathcal{R}_{l}\left(\left(\partial_{m} u_{l}\right) \Delta_{j} u_{k}\right) \Delta_{j} u_{m} d x \\
& -\int \mathcal{R}_{k} \mathcal{R}_{l}\left(\left(\partial_{m} u_{k}\right) \Delta_{j} u_{l}\right) \Delta_{j} u_{m} d x .
\end{aligned}
$$

It is then clear that

$$
\left|\int I I I_{2} \cdot \Delta_{j} u d x\right| \leq c\|\nabla u\|_{L^{\infty}}\left\|\Delta_{j} u\right\|_{L^{2}}^{2} .
$$

(3.7) is obtained by combining (3.8), (3.9) and (3.10). This completes the proof of Lemma 3.3.

We now resume the proof of Proposition 3.1. Collecting the estimates in (3.5), (3.6) and (3.7), we obtain

$$
\frac{d}{d t}\left\|\Delta_{j} u\right\|_{L^{2}}+c \nu 2^{2 \alpha j}\left\|\Delta_{j} u\right\|_{L^{2}} \leq c\|\nabla u\|_{L^{\infty}}\left\|\Delta_{j} u\right\|_{L^{2}}
$$


Multiplying by $2^{j r}$ and taking $\sup _{j}$ leads to

$$
\frac{d}{d t}\|u\|_{B_{2, \infty}^{r}}+c \nu\|u\|_{B_{2, \infty}^{r+2 \alpha}} \leq c\|\nabla u\|_{L^{\infty}}\|u\|_{B_{2, \infty}^{r}} .
$$

Note that we have switched $\frac{d}{d t}$ and $\sup _{j}$. This can be justified using the Monotone Convergence Theorem. Finally, we apply Proposition A.1 to bound $\|\nabla u\|_{L^{\infty}}$ in terms of $\|u\|_{B_{2, \infty}^{s}}$ with $s>1+\frac{d}{2}$, namely

$$
\|\nabla u\|_{L^{\infty}} \leq c\|\nabla u\|_{B_{2, \infty}^{s-1}} \leq c\|u\|_{B_{2, \infty}^{s}} .
$$

Inserting (3.12) in (3.11) finishes the proof.

Proof of Theorem 3.2. We apply the method of successive approximation. It consists of constructing a successive approximation sequence $\left\{\left(u^{(n)}, P^{(n)}\right)\right\}$ and showing its convergence to $(u, P)$, the solution of the IVP $(3.1)$.

Consider a successive approximation sequence $\left\{\left(u^{(n)}, P^{(n)}\right)\right\}$ satisfying

$$
\left\{\begin{array}{l}
u^{(0)}=0, P^{(0)}=0 \\
\partial_{t} u^{(n+1)}+u^{(n)} \cdot \nabla u^{(n+1)}=-\nabla P^{(n+1)}-\nu(-\Delta)^{\alpha} u^{(n+1)} \\
\nabla \cdot u^{(n+1)}=0 \\
u^{(n+1)}(x, 0)=u_{0}^{(n+1)}(x)=S_{n+1} u_{0}(x) .
\end{array}\right.
$$

To show that $\left\{\left(u^{(n)}, P^{(n)}\right)\right\}$ converges, we prove that

i) $\left\{\left(u^{(n)}, P^{(n)}\right)\right\}$ is bounded uniformly in

$$
\left(L^{\infty}\left([0, \infty) ; B_{2, \infty}^{r}\right) \cap L^{1}\left([0, \infty) ; B_{2, \infty}^{r+2 \alpha}\right)\right) \times L^{1}\left([0, \infty) ; B_{2, \infty}^{r}\right) ;
$$

ii) $\left\{\left(u^{(n)}, P^{(n)}\right)\right\}$ is a Cauchy sequence in

$$
\left(L^{\infty}\left([0, \infty) ; B_{2, \infty}^{r-1}\right) \cap L^{1}\left([0, \infty) ; B_{2, \infty}^{r-1+2 \alpha}\right)\right) \times L^{1}\left([0, \infty) ; B_{2, \infty}^{r-1}\right) .
$$

To establish i), we proceed as in the proof of Proposition 3.1. That is, we start with the second equation in (3.13) and estimate $u^{(n+1)}$ in $B_{2, \infty}^{r}$. We deal with the term involving $P^{(n+1)}$ as in Lemma 3.7. It is bounded by

$$
\left\|\Delta_{j} \nabla P^{(n+1)}\right\|_{L^{2}} \leq c\left(\left\|\nabla u^{(n)}\right\|_{L^{\infty}}\left\|\Delta_{j} u^{(n+1)}\right\|_{L^{2}}+\left\|\nabla u^{(n+1)}\right\|_{L^{\infty}}\left\|\Delta_{j} u^{(n)}\right\|_{L^{2}}\right) .
$$

After going through the steps as in proof of Proposition 3.1, we arrive at

$$
\begin{aligned}
& \frac{d}{d t}\left\|u^{(n+1)}\right\|_{B_{2, \infty}^{r}}+c \nu\left\|u^{(n+1)}\right\|_{B_{2, \infty}^{r+2 \alpha}} \\
& \quad \leq c\left(\left\|\nabla u^{(n)}\right\|_{L^{\infty}}\left\|u^{(n+1)}\right\|_{B_{2, \infty}^{r}}+\left\|\nabla u^{(n+1)}\right\|_{L^{\infty}}\left\|u^{(n)}\right\|_{B_{2, \infty}^{r}}\right) .
\end{aligned}
$$

Since $r+2 \alpha>1+\frac{d}{2}$, we apply Proposition A.1 to bound $\left\|\nabla u^{(n)}\right\|_{L^{\infty}}$ and $\left\|\nabla u^{(n+1)}\right\|_{L^{\infty}}$. Therefore,

$$
\begin{aligned}
& \frac{d}{d t}\left\|u^{(n+1)}\right\|_{B_{2, \infty}^{r}}+c \nu\left\|u^{(n+1)}\right\|_{B_{2, \infty}^{r+2 \alpha}} \\
& \quad \leq c\left(\left\|u^{(n)}\right\|_{B_{2, \infty}^{r+2 \alpha}}\left\|u^{(n+1)}\right\|_{B_{2, \infty}^{r}}+\left\|u^{(n+1)}\right\|_{B_{2, \infty}^{r+2 \alpha}}\left\|u^{(n)}\right\|_{B_{2, \infty}^{r}}\right) .
\end{aligned}
$$

This inequality allows us to show inductively that if (3.3) holds, namely

$$
\left\|u_{0}\right\|_{B_{2, \infty}^{r}} \leq C_{0} \nu
$$


then for any $t>0$,

$$
\sup _{\tau \in[0, t]}\left\|u^{(n)}(\cdot, \tau)\right\|_{B_{2, \infty}^{r}}+c \nu \int_{0}^{t}\left\|u^{(n)}(\cdot, \tau)\right\|_{B_{2, \infty}^{r+2 \alpha}} d \tau \leq 2 C_{0} \nu .
$$

Thus $\left\{u^{(n)}\right\}$ is bounded uniformly in $L^{\infty}\left([0, \infty) ; B_{2, \infty}^{r}\right) \cap L^{1}\left([0, \infty) ; B_{2, \infty}^{r+2 \alpha}\right)$. To see the uniform boundedness of $\left\{P^{(n)}\right\}$ in $L^{1}\left([0, \infty) ; B_{2, \infty}^{r}\right)$, we note that

$$
P^{(n+1)}=\mathcal{R}_{k} \mathcal{R}_{l}\left(u_{k}^{(n)} u_{l}^{(n+1)}\right) .
$$

We then apply Proposition A.4 and Proposition A.1 to obtain

$$
\begin{aligned}
\left\|P^{(n+1)}\right\|_{B_{2, \infty}^{r}} & \leq \sum_{k, l=1}^{d}\left\|u_{k}^{(n)} u_{l}^{(n+1)}\right\|_{B_{2, \infty}^{r}} \\
& \leq c\left(\left\|u^{(n)}\right\|_{L^{\infty}}\left\|u^{(n+1)}\right\|_{B_{2, \infty}^{r}}+\left\|u^{(n+1)}\right\|_{L^{\infty}}\left\|u^{(n)}\right\|_{B_{2, \infty}^{r}}\right) \\
& \leq c\left(\left\|u^{(n)}\right\|_{B_{2, \infty}^{r+2 \alpha}}\left\|u^{(n+1)}\right\|_{B_{2, \infty}^{r}}+\left\|u^{(n+1)}\right\|_{B_{2, \infty}^{r+2 \alpha}}\left\|u^{(n)}\right\|_{B_{2, \infty}^{r}}\right) .
\end{aligned}
$$

It then follows from (3.15) that for a constant $c$,

$$
\left\|P^{(n)}\right\|_{L^{1}\left([0, \infty) ; B_{2, \infty}^{r}\right)} \leq c \nu .
$$

This completes the proof of i).

To establish ii), we consider the differences

$$
v^{(n+1)}=u^{(n+1)}-u^{(n)}, \quad Q^{(n+1)}=P^{(n+1)}-P^{(n)},
$$

which satisfy

$$
\left\{\begin{array}{l}
\partial_{t} v^{(n+1)}+u^{(n)} \cdot \nabla v^{(n+1)}+\nu \Lambda^{2 \alpha} v^{(n+1)}=-\nabla Q^{(n+1)}+v^{(n)} \cdot \nabla u^{(n)} \\
\nabla \cdot v^{(n+1)}=0 \\
v^{(n+1)}(x, 0)=v_{0}^{(n+1)}(x)=\Delta_{n+1} u_{0}
\end{array}\right.
$$

We shall show that for any integer $n>0$

$$
\sup _{\tau \in[0, t]}\left\|v^{(n)}(\cdot, \tau)\right\|_{B_{2, \infty}^{r-1}}+c \nu \int_{0}^{t}\left\|v^{(n)}(\cdot, \tau)\right\|_{B_{2, \infty}^{r-1+2 \alpha}} d \tau \leq\left\|u_{0}\right\|_{B_{2, \infty}^{r}} 2^{-(n-3)}
$$

valid for any $t>0$. To establish (3.17), we estimate $\left\{v^{(n)}\right\}$ in $B_{2, \infty}^{r-1}$. After going through a similar procedure as above, we obtain

$$
\begin{aligned}
& \frac{d}{d t}\left\|v^{(n+1)}\right\|_{B_{2, \infty}^{r-1}}+c \nu\left\|v^{(n+1)}\right\|_{B_{2, \infty}^{r-1+2 \alpha}} \leq\left\|\nabla Q^{(n+1)}\right\|_{B_{2, \infty}^{r-1}} \\
& \quad+\left\|v^{(n)} \cdot \nabla u^{(n)}\right\|_{B_{2, \infty}^{r-1}}+c \sup _{j} 2^{(r-1) j}\left\|\left[u^{(n)} \cdot \nabla, \Delta_{j}\right] v^{(n+1)}\right\|_{L^{2}} .
\end{aligned}
$$

To obtain suitable bounds for the terms on the right-hand side, we apply the commutator estimate in Proposition A.3.

$$
\begin{aligned}
& \sup _{j} 2^{(r-1) j}\left\|\left[u^{(n)} \cdot \nabla, \Delta_{j}\right] v^{(n+1)}\right\|_{L^{2}} \\
& \leq c\left(\left\|\nabla u^{(n)}\right\|_{L^{\infty}}\left\|v^{(n+1)}\right\|_{B_{2, \infty}^{r-1}}+\left\|v^{(n+1)}\right\|_{L^{\infty}}\left\|u^{(n)}\right\|_{B_{2, \infty}^{r}}\right) .
\end{aligned}
$$

The term involving $\nabla Q^{(n+1)}$ can be estimated similarly as in (3.14), but we apply the commutator estimate in Proposition A.3 rather than the one in Proposition A.2.

$$
\left\|\nabla Q^{(n+1)}\right\|_{B_{2, \infty}^{r-1}} \leq c\left(\left\|\nabla u^{(n)}\right\|_{L^{\infty}}\left\|v^{(n+1)}\right\|_{B_{2, \infty}^{r-1}}+\left\|v^{(n+1)}\right\|_{L^{\infty}}\left\|u^{(n)}\right\|_{B_{2, \infty}^{r}}\right) .
$$


Since $r>1$, we apply Proposition A.4 to bound the product $v^{(n)} \cdot \nabla u^{(n)}$,

$$
\left\|v^{(n)} \cdot \nabla u^{(n)}\right\|_{B_{2, \infty}^{r-1}} \leq c\left(\left\|v^{(n)}\right\|_{L^{\infty}}\left\|\nabla u^{(n)}\right\|_{B_{2, \infty}^{r-1}}+\left\|v^{(n)}\right\|_{B_{2, \infty}^{r-1}}\left\|\nabla u^{(n)}\right\|_{L^{\infty}}\right)
$$

We further apply Proposition A.1 to bound the $L^{\infty}$-norms in (3.19), (3.20) and (3.21) and then insert the resulting estimates in (3.18). This leads us to the inequality

$$
\begin{aligned}
\frac{d}{d t}\left\|v^{(n+1)}\right\|_{B_{2, \infty}^{r-1}}+c \nu\left\|v^{(n+1)}\right\|_{B_{2, \infty}^{r-1+2 \alpha}} & \\
\leq & c\left(\left\|u^{(n)}\right\|_{B_{2, \infty}^{r+2 \alpha}}\left\|v^{(n+1)}\right\|_{B_{2, \infty}^{r-1}}+\left\|v^{(n+1)}\right\|_{B_{2, \infty}^{r-1+2 \alpha}}\left\|u^{(n)}\right\|_{B_{2, \infty}^{r}}\right) \\
& +c\left(\left\|v^{(n)}\right\|_{B_{2, \infty}^{r-1+2 \alpha}}\left\|u^{(n)}\right\|_{B_{2, \infty}^{r}}+\left\|v^{(n)}\right\|_{B_{2, \infty}^{r-1}}\left\|u^{(n)}\right\|_{B_{2, \infty}^{r+2 \alpha}} .\right.
\end{aligned}
$$

Integrating this inequality over $[0, t]$, we obtain

$$
\begin{aligned}
\sup _{\tau \in[0, t]} & \left\|v^{(n+1)}(\cdot, \tau)\right\|_{B_{2, \infty}^{r-1}}+c \nu \int_{0}^{t}\left\|v^{(n+1)}(\cdot, \tau)\right\|_{B_{2, \infty}^{r-1+2 \alpha}} d \tau \\
\leq & \left\|\Delta_{n+1} u_{0}\right\|_{B_{2, \infty}^{r-1}}+c\left(\sup _{\tau \in[0, t]}\left\|v^{(n+1)}\right\|_{B_{2, \infty}^{r-1}}+\sup _{\tau \in[0, t]}\left\|v^{(n)}\right\|_{B_{2, \infty}^{r-1}}\right) \\
& \int_{0}^{t}\left\|u^{(n)}\right\|_{B_{2, \infty}^{r+2 \alpha}} d \tau \\
& +\sup _{\tau \in[0, t]}\left\|u^{(n)}\right\|_{B_{2, \infty}^{r}} \int_{0}^{t}\left(\left\|v^{(n)}\right\|_{B_{2, \infty}^{r-1+2 \alpha}}+\left\|v^{(n+1)}\right\|_{B_{2, \infty}^{r-1+2 \alpha}}\right) d \tau .
\end{aligned}
$$

Noticing that $\left\|\Delta_{n+1} u_{0}\right\|_{B_{2, \infty}^{r-1}} \leq\left\|u_{0}\right\|_{B_{2, \infty}^{r}} 2^{-n}$, (3.22) allows us to prove by induction that (3.17) holds. As a consequence, we have shown that $\left\{u^{(n)}\right\}$ is a Cauchy sequence in

$$
L^{\infty}\left([0, \infty) ; B_{2, \infty}^{r-1}\right) \cap L^{1}\left([0, \infty) ; B_{2, \infty}^{r-1+2 \alpha}\right) .
$$

The bound for $\left\{Q^{(n)}\right\}$ can be obtained in a similar fashion as for $\left\{P^{(n)}\right\}$. According to $(3.16)$,

$$
Q^{(n+1)}=\mathcal{R}_{k} \mathcal{R}_{l}\left(u_{k}^{(n)} v_{l}^{(n+1)}+v_{k}^{(n)} u_{l}^{(n)}\right) .
$$

Therefore,

$$
\begin{gathered}
\left\|Q^{(n+1)}(\cdot, t)\right\|_{B_{2, \infty}^{r-1}} \leq c\left(\left(\left\|v^{(n)}\right\|_{B_{2, \infty}^{r-1}}+\left\|v^{(n+1)}\right\|_{B_{2, \infty}^{r-1}}\right)\left\|u^{(n)}\right\|_{B_{2, \infty}^{r-1+2 \alpha}}\right. \\
+c\left(\left(\left\|v^{(n)}\right\|_{B_{2, \infty}^{r-1+2 \alpha}}+\left\|v^{(n+1)}\right\|_{B_{2, \infty}^{r-1+2 \alpha}}\right)\left\|u^{(n)}\right\|_{B_{2, \infty}^{r-1}}\right.
\end{gathered}
$$

Since $\left\{u^{(n)}\right\}$ is bounded uniformly in $L^{\infty}\left([0, \infty) ; B_{2, \infty}^{r}\right) \cap L^{1}\left([0, \infty) ; B_{2, \infty}^{r+2 \alpha}\right)$ and $\left\{v^{(n)}\right\}$ in $L^{\infty}\left([0, \infty) ; B_{2, \infty}^{r-1}\right) \cap L^{1}\left([0, \infty) ; B_{2, \infty}^{r-1+2 \alpha}\right)$, we obtain that $\left\{Q^{(n)}\right\}$ is bounded uniformly in $L^{1}\left([0, \infty) ; B_{2, \infty}^{r-1}\right)$. That is, $\left\{P^{(n)}\right\}$ is a Cauchy sequence in $L^{1}\left([0, \infty) ; B_{2, \infty}^{r-1}\right)$. This completes the proof of ii).

We can now conclude from ii) that there exists a unique

$$
(u, P) \in\left(L^{\infty}\left([0, \infty) ; B_{2, \infty}^{r-1}\right) \cap L^{1}\left([0, \infty) ; B_{2, \infty}^{r-1+2 \alpha}\right)\right) \times L^{1}\left([0, \infty) ; B_{2, \infty}^{r-1}\right)
$$

such that

$$
\begin{aligned}
u^{(n)} \rightarrow u \quad & \text { in } L^{\infty}\left([0, \infty) ; B_{2, \infty}^{r-1}\right) \cap L^{1}\left([0, \infty) ; B_{2, \infty}^{r-1+2 \alpha}\right), \\
& P^{(n)} \rightarrow P \quad \text { in } L^{1}\left([0, \infty) ; B_{2, \infty}^{r-1}\right) .
\end{aligned}
$$


Because of i), $(u, P)$ actually belongs to

$$
\left(L^{\infty}\left([0, \infty) ; B_{2, \infty}^{r}\right) \cap L^{1}\left([0, \infty) ; B_{2, \infty}^{r+2 \alpha}\right)\right) \times L^{1}\left([0, \infty) ; B_{2, \infty}^{r}\right) .
$$

In addition, $\left\{u^{(n)}\right\}$ and $u$ are both absolutely continuous from $[0, \infty)$ to $B_{2, \infty}^{r-1}$, or simply $u^{(n)}, u \in C\left([0, \infty) ; B_{2, \infty}^{r-1}\right)$. To prove this fact, we rewrite the second equation in (3.13) in the integral form,

$$
u^{(n+1)}(x, t)=u_{0}^{(n+1)}(x)+\int_{0}^{t} g^{(n+1)}(x, \tau) d \tau
$$

with

$$
g^{(n+1)}=-u^{(n)} \cdot \nabla u^{(n+1)}-\nabla P^{(n+1)}-\nu(-\Delta)^{\alpha} u^{(n+1)} .
$$

Since $g^{(n+1)}$ has the following bound

$$
\begin{aligned}
\left\|g^{(n+1)}(\cdot, t)\right\|_{B_{2, \infty}^{r-1}} \leq & c\left(\left\|u^{(n)}\right\|_{B_{2, \infty}^{r-1+2 \alpha}}\left\|u^{(n+1)}\right\|_{B_{2, \infty}^{r}}+\left\|u^{(n)}\right\|_{\left.B_{2, \infty}^{r-1}\left\|u^{(n+1)}\right\|_{B_{2, \infty}^{r+2 \alpha}}\right)}\right. \\
& +\left\|\nabla P^{(n+1)}\right\|_{B_{2, \infty}^{r-1}}+\nu\left\|u^{(n+1)}\right\|_{B_{2, \infty}^{r-1+2 \alpha}}
\end{aligned}
$$

and each term on the right is in $L^{1}([0, \infty))$, we have

$$
\left\|g^{(n+1)}(\cdot, t)\right\|_{B_{2, \infty}^{r-1}} \in L^{1}([0, \infty)) .
$$

Therefore, $\left\{u^{(n+1)}\right\}$ is absolutely continuous from $[0, \infty)$ to $B_{2, \infty}^{r-1}$ and so is $u$.

Finally, letting $n \rightarrow \infty$ in (3.13), we obtain that $(u, P)$ satisfies the GNS equations in (3.1). This completes the proof of Theorem 3.2.

\section{4. $\dot{B}_{2,1}^{r}$ and $B_{2, q}^{r}$ Solutions}

We continue in this section the study of solutions of the IVP (3.1), but we now assume that $u_{0}$ is either in $\dot{B}_{2,1}^{r}$ or in $B_{2, q}^{r}$ with $q \in[1, \infty)$. The major results are presented in three theorems. The first theorem is on solutions in the homogeneous Besov space $\dot{B}_{2,1}^{r}$ while the second one is on solutions in the inhomogeneous space $B_{2,1}^{r}$. The third theorem concerns solutions in $B_{2, q}^{r}$ with $1<q<\infty$. It appears that the conclusion in the third theorem is invalid for $\dot{B}_{2, q}^{r}$.

We first state the theorem for $u_{0} \in \dot{B}_{2,1}^{r}$.

Theorem 4.1. Consider the solutions of the IVP (3.1) with $\nu>0$ and $\alpha<$ $\frac{1}{2}+\frac{d}{4}$. If $u_{0} \in \dot{B}_{2,1}^{r}$ with

and

$$
r=1+\frac{d}{2}-2 \alpha>1
$$

for some suitable constant $C_{1}$, then the IVP (3.1) has a unique global solution $(u, P)$ satisfying

$$
\begin{gathered}
u \in L^{\infty}\left([0, \infty) ; \dot{B}_{2,1}^{r}\right) \cap L^{1}\left([0, \infty) ; \dot{B}_{2,1}^{r+2 \alpha}\right) \cap C\left([0, \infty) ; \dot{B}_{2,1}^{r-1}\right), \\
P \in L^{1}\left([0, \infty) ; \dot{B}_{2,1}^{r}\right)
\end{gathered}
$$


and

$$
\|u(\cdot, t)\|_{\dot{B}_{2,1}^{r}} \leq 2 C_{1} \nu, \quad \text { for any } t>0
$$

A similar result holds for $u_{0}$ in the inhomogeneous Besov space $B_{2,1}^{r}$, but the condition on $r$ can be relaxed to $r \geq 1+\frac{d}{2}-2 \alpha$.

TheOrem 4.2. Consider the solutions of (3.1) with $\nu>0$ and $\alpha<\frac{1}{2}+\frac{d}{4}$. If $u_{0} \in B_{2,1}^{r}$ with

and

$$
r>1, \quad r \geq 1+\frac{d}{2}-2 \alpha
$$

$$
\left\|u_{0}\right\|_{B_{2,1}^{r}} \leq C_{2} \nu
$$

for some suitable constant $C_{2}$, then the IVP (3.1) has a unique global solution $u$ satisfying

$$
\begin{gathered}
u \in L^{\infty}\left([0, \infty) ; B_{2,1}^{r}\right) \cap L^{1}\left([0, \infty) ; B_{2,1}^{r+2 \alpha}\right) \cap C\left([0, \infty) ; B_{2,1}^{r-1}\right), \\
P \in L^{1}\left([0, \infty) ; B_{2,1}^{r}\right)
\end{gathered}
$$

and

$$
\|u(\cdot, t)\|_{B_{2,1}^{r}} \leq 2 C_{2} \nu \quad \text { for any } t>0 .
$$

Theorem 4.3. If $u_{0} \in B_{2, q}^{r}$ with

$$
1<q<\infty, \quad r>1, \quad r>1+\frac{d}{2}-\frac{2 \alpha}{q}
$$

and

$$
\left\|u_{0}\right\|_{B_{2, q}^{r}} \leq C_{3} \nu
$$

for some suitable constant $C_{3}$, then the IVP (3.1) has a unique global solution $(u, P)$ satisfying

and

$$
\begin{gathered}
u \in L^{\infty}\left([0, \infty) ; B_{2, q}^{r}\right) \cap L^{q}\left([0, \infty) ; B_{2, q}^{r+\frac{2 \alpha}{q}}\right) \cap C\left([0, \infty) ; B_{2, \infty}^{r-1}\right) . \\
P \in L^{q}\left([0, \infty) ; B_{2, q}^{r}\right)
\end{gathered}
$$

$$
\|u(\cdot, t)\|_{B_{2, q}^{r}} \leq 2 C_{3} \nu \quad \text { for any } t>0
$$

We now prove these theorems.

Proof of Theorem 4.1. The major tool is the method of successive approximation. Since the details resemble those in the proof of Theorem 3.2, it is redundant to provide a full proof of this theorem. Instead, we prove a major a priori estimate, which can be easily extended into a complete proof of Theorem 4.1. As in the proof of Proposition 3.1, we have

$$
\frac{d}{d t}\left\|\Delta_{j} u\right\|_{L^{2}}+c \nu 2^{2 \alpha j}\left\|\Delta_{j} u\right\|_{L^{2}} \leq c\|\nabla u\|_{L^{\infty}}\left\|\Delta_{j} u\right\|_{L^{2}} .
$$

Multiplying (4.5) by $2^{j r}$ and summing over $j \in \mathbb{Z}$ yields

$$
\frac{d}{d t}\|u\|_{\dot{B}_{2,1}^{r}}+c \nu\|u\|_{\dot{B}_{2,1}^{r+2 \alpha}} \leq c\|\nabla u\|_{L^{\infty}}\|u\|_{\dot{B}_{2,1}^{r}} .
$$


Since $r+2 \alpha=1+\frac{d}{2}$, we have according to 1) of Proposition A.1,

$$
\|\nabla u\|_{L^{\infty}} \leq c\|\nabla u\|_{\dot{B}_{2,1}^{r+2 \alpha-1}} \leq c\|u\|_{\dot{B}_{2,1}^{r+2 \alpha}} .
$$

This leads to the inequality

$$
\frac{d}{d t}\|u\|_{\dot{B}_{2,1}^{r}}+c \nu\|u\|_{\dot{B}_{2,1}^{r+2 \alpha}} \leq c\|u\|_{\dot{B}_{2,1}^{r+2 \alpha}}\|u\|_{\dot{B}_{2,1}^{r}} .
$$

That is, for some suitable constant $C_{1}>0$,

$$
\frac{d}{d t}\|u\|_{\dot{B}_{2,1}^{r}} \leq-c\left(C_{1} \nu-\|u\|_{\dot{B}_{2,1}^{r}}\right)\|u\|_{\dot{B}_{2,1}^{r+2 \alpha}} .
$$

If $u_{0}$ satisfies (4.1), this inequality then implies that $\|u(\cdot, t)\|_{\dot{B}_{2,1}^{r}}$ is a non-increasing function of $t$ for $t>0$. This yields the boundedness of $u$ in $L^{\infty}\left([0, \infty) ; \dot{B}_{2,1}^{r}\right) \cap$ $L^{1}\left([0, \infty) ; \dot{B}_{2,1}^{r+2 \alpha}\right)$. To establish an a priori estimate for $P$, we note that

$$
P=-\mathcal{R}_{k} \mathcal{R}_{l}\left(u_{k}, u_{l}\right) .
$$

Applying Proposition A.4 and Proposition A.1 yields

$$
\|P(\cdot, t)\|_{B_{2,1}^{r}} \leq c\|u\|_{\dot{B}_{2,1}^{r-1+2 \alpha}}\|u\|_{\dot{B}_{2,1}^{r}} .
$$

Therefore, $\|P(\cdot, t)\|_{\dot{B}_{2,1}^{r}}$ is in $L^{1}([0, \infty))$ or $P \in L^{1}\left([0, \infty) ; \dot{B}_{2,1}^{r}\right)$. As explained at the beginning of this proof, we omit further details. This concludes the proof.

Proof of Theorem 4.2. The proof is similar to that of Theorem 4.1. The major difference is that here we use part 2) of Proposition A.1 to bound $\|\nabla u\|_{L^{\infty}}$, namely

$$
\|\nabla u\|_{L^{\infty}} \leq c\|u\|_{B_{2,1}^{s}} .
$$

This inequality is valid for any $s \geq 1+\frac{d}{2}$ and thus allows the condition on $r$ to be relaxed to $r \geq 1+\frac{d}{2}-2 \alpha$. We shall again omit the details on constructing a successive approximation sequence and showing its convergence to the solution of the GNS equations.

Proof of Theorem 4.3. As we have explained previously, it suffices to present only relevant a priori estimates. For $q>1$, we multiply (4.5) by $q 2^{q r j}\left\|\Delta_{j} u\right\|_{L^{p}}^{q-1}$ and then sum over $j$ from -1 to $\infty$ to obtain

$$
\frac{d}{d t}\|u\|_{B_{2, q}^{r}}^{q}+c q \nu\|u\|_{B_{2, q}^{r+2 \alpha / q}}^{q} \leq c\|\nabla u\|_{L^{\infty}}\|u\|_{B_{2, q}^{r}}^{q} .
$$

Since $\left.r+\frac{2 \alpha}{q}>1+\frac{d}{2}, 3\right)$ of Proposition A.1 implies that

$$
\|\nabla u\|_{L^{\infty}} \leq c\|\nabla u\|_{B_{2, q}^{r+\frac{2 \alpha}{q}-1}} \leq c\|u\|_{B_{2, q}^{r+\frac{2 \alpha}{q}}} .
$$

Inserting this inequality and the basic embedding inequality

$$
\|u\|_{B_{2, q}^{r}} \leq\|u\|_{B_{2, q}^{r+2 \alpha / q}}
$$

in (4.6) yields

$$
\frac{d}{d t}\|u\|_{B_{2, q}^{r}}^{q} \leq-c\left(C_{3} \nu-\|u\|_{B_{2, q}^{r}}\right)\|u\|_{B_{2, q}^{r+2 \alpha / q}}^{q},
$$

where $C_{3}$ is a suitable constant depending on $\alpha, q$ and $r$ only. This differential inequality implies that $\|u(\cdot, t)\|_{B_{2, q}^{r}}$ is a non-increasing function of $t \geq 0$. Thus, if 
$u_{0}$ satisfies (4.3), then (4.4) holds for all $t>0$. An a priori estimate for $P$ can be obtained as in the proof of Theorem 4.1. This completes the proof of Theorem 4.3.

\section{Scaling invariance}

In this section, we examine some properties of the Besov spaces in which the solutions of the GNS equations have been studied. In particular, we investigate the scaling invariance property of these spaces and their implications. This will help us have a better understanding of the results presented in the previous two sections.

The Besov spaces $\dot{B}_{2, \infty}^{1+\frac{d}{2}-2 \alpha}$ and $\dot{B}_{2,1}^{1+\frac{d}{2}-2 \alpha}$ are critical to solutions of the NavierStokes equations. As mentioned in the introduction, solutions of the GNS equations obey a scaling property. That is, if $(u, P)$ satisfies the GNS equations (1.1), then $\left(u_{\lambda}, P_{\lambda}\right)$ also satisfies $(1.1)$, where

$$
u_{\lambda}(x, t)=\lambda^{2 \alpha-1} u\left(\lambda x, \lambda^{2 \alpha} t\right), \quad P_{\lambda}(x, t)=\lambda^{4 \alpha-2} P\left(\lambda x, \lambda^{2 \alpha} t\right) .
$$

The Besov spaces $\dot{B}_{2, \infty}^{1+\frac{d}{2}-2 \alpha}$ and $\dot{B}_{2,1}^{1+\frac{d}{2}-2 \alpha}$ are critical in the sense that the norm of $u_{\lambda}$ is essentially invariant in these spaces. More precisely, we have the following lemma.

LEMMA 5.1. If $\lambda=2^{k}$ for some $k \in \mathbb{Z}$, then

$$
\begin{gathered}
\left\|u_{\lambda}(\cdot, t)\right\|_{\dot{B}_{2, \infty}^{1+\frac{d}{2}-2 \alpha}}=\left\|u\left(\cdot, 2^{2 \alpha k} t\right)\right\|_{\dot{B}_{2, \infty}^{1+\frac{d}{2}-2 \alpha}}, \\
\left\|u_{\lambda}(\cdot, t)\right\|_{\dot{B}_{2,1}^{1+\frac{d}{2}-2 \alpha}}=\left\|u\left(\cdot, 2^{2 \alpha k} t\right)\right\|_{\dot{B}_{2,1}^{1+\frac{d}{2}-2 \alpha}},
\end{gathered}
$$

More generally, for any $\lambda>0$,

$$
\begin{aligned}
&\left\|u_{\lambda}(\cdot, t)\right\|_{\dot{B}_{2, \infty}^{1+\frac{d}{2}-2 \alpha}} \approx\left\|u\left(\cdot, 2^{2 \alpha k} t\right)\right\|_{\dot{B}_{2, \infty}^{1+\frac{d}{2}-2 \alpha}}, \\
&\left\|u_{\lambda}(\cdot, t)\right\|_{\dot{B}_{2,1}^{1+\frac{d}{2}-2 \alpha}} \approx\left\|u\left(\cdot, 2^{2 \alpha k} t\right)\right\|_{\dot{B}_{2,1}^{1+\frac{d}{2}-2 \alpha}},
\end{aligned}
$$

where $\approx$ denotes the equivalence of two norms.

Proof. For any $j \in \mathbb{Z}$, we have

$$
\begin{aligned}
\left\|\Delta_{j} u_{\lambda}(\cdot, t)\right\|_{L^{2}}^{2} & =\int_{\mathbb{R}^{d}} \widehat{\Phi}_{j}(\xi)^{2}\left|\widehat{u}_{\lambda}(\xi)\right|^{2} d \xi \\
& =\lambda^{4 \alpha-2-d} \int_{\mathbb{R}^{d}} \widehat{\Phi}_{0}\left(2^{-j} \lambda \xi\right)^{2}\left|\widehat{u}\left(\xi, \lambda^{2 \alpha} t\right)\right|^{2} d \xi .
\end{aligned}
$$

When $\lambda=2^{k}$, this equality implies

$$
\left\|\Delta_{j} u_{\lambda}(\cdot, t)\right\|_{L^{2}}=2^{k(2 \alpha-1-d / 2)}\left\|\Delta_{j-k} u\left(\cdot, 2^{2 \alpha k} t\right)\right\|_{L^{2}} .
$$

Thus,

$$
\begin{aligned}
\left\|u_{\lambda}(\cdot, t)\right\|_{\dot{B}_{2, \infty}^{1+\frac{d}{2}-2 \alpha}} & =\sup _{-\infty<j<\infty} 2^{\left(1+\frac{d}{2}-2 \alpha\right) j}\left\|\Delta_{j} u_{\lambda}(\cdot, t)\right\|_{L^{2}} \\
& =\sup _{-\infty<j<\infty} 2^{\left(1+\frac{d}{2}-2 \alpha\right)(j-k)}\left\|\Delta_{j-k} u\left(\cdot, 2^{2 \alpha k} t\right)\right\|_{L^{2}} \\
& =\left\|u\left(\cdot, 2^{2 \alpha k} t\right)\right\|_{\dot{B}_{2, \infty}^{1+\frac{d}{2}-2 \alpha}}
\end{aligned}
$$


This proves (5.1). For a general $\lambda>0$, choose $k \in \mathbb{Z}$ such that

$$
2^{k-1}<\lambda \leq 2^{k}
$$

Since $\widehat{\Phi}_{0}$ can be chosen to satisfy

$$
\widehat{\Phi}_{0}\left(\frac{\xi}{2^{j-k}}\right) \leq \widehat{\Phi}_{0}\left(\frac{\lambda \xi}{2^{j}}\right) \leq \widehat{\Phi}_{0}\left(\frac{\xi}{2^{j-k+1}}\right),
$$

we obtain by inserting these inequalities in (5.4)

$$
\lambda^{2 \alpha-1-\frac{d}{2}}\left\|\Delta_{j-k} u\left(\cdot, 2^{2 \alpha k} t\right)\right\|_{L^{2}} \leq\left\|\Delta_{j} u_{\lambda}(\cdot, t)\right\|_{L^{2}} \leq \lambda^{2 \alpha-1-\frac{d}{2}}\left\|\Delta_{j-k+1} u\left(\cdot, 2^{2 \alpha k} t\right)\right\|_{L^{2}} .
$$

(5.3) is then established after following the lines as in the case $\lambda=2^{k}$. The proof of (5.2) is similar to that of (5.1) and the difference is that one replaces $\sup _{j}$ by $\sum_{j \in \mathbb{Z}}$ in (5.5). This completes the proof.

Now, we explore some of the implications of the scaling invariance of these spaces. Theorem 3.2 asserts that the GNS equations have a unique global solution corresponding to any initial datum $u_{0}$ in the inhomogeneous Besov space $B_{2, \infty}^{r}$ and comparable to $\nu$, where $r>1+\frac{d}{2}-2 \alpha$. If the conclusion of Theorem 3.2 were also true for the homogeneous Besov space $\dot{B}_{2, \infty}^{r}$ with $r>1+\frac{d}{2}-2 \alpha$, then one would be able to remove the smallness condition that $u_{0}$ is comparable to $\nu$ in $\dot{B}_{2, \infty}^{r}$. The reason is simple. For any $u_{0} \in \dot{B}_{2, \infty}^{r}$,

$$
u_{0 \lambda}(x, t) \equiv \lambda^{2 \alpha-1} u_{0}\left(\lambda x, \lambda^{2 \alpha} t\right)
$$

remains in $\dot{B}_{2, \infty}^{r}$ and is comparable to $\nu$ in $\dot{B}_{2, \infty}^{r}$ for sufficiently small $\lambda>0$ since

$$
\left\|u_{0 \lambda}\right\|_{\dot{B}_{2, \infty}^{r}} \approx \lambda^{r-\left(1+\frac{d}{2}-2 \alpha\right)}\left\|u_{0}\right\|_{\dot{B}_{2, \infty}^{r}}
$$

Then $u_{\lambda}$ emanating from $u_{0 \lambda}$ leads to $u$, the solution corresponding to $u_{0}$.

Similarly, the invariance property of $\dot{B}_{2,1}^{1+\frac{d}{2}-2 \alpha}$ may provide another explanation as to why Theorem 4.2 allows for solutions in any Besov space $B_{2,1}^{r}$ with $r \geq$ $1+\frac{d}{2}-2 \alpha$ but the result in Theorem 4.1 is only for $\dot{B}_{2,1}^{1+\frac{d}{2}-2 \alpha}$.

\section{Appendix}

As mentioned before, this appendix contains several inequalities that we have used to prove our major results. First, we present the logarithmic Besov type inequalities. The inequalities allows us to bound the $L^{\infty}$-norm in terms of the norms in Besov spaces.

Proposition A.1. Let $1 \leq p \leq \infty$.

1) If $f \in \dot{B}_{p, 1}^{r}\left(\mathbb{R}^{d}\right)$ with $r=\frac{d}{p}$, then

$$
\|f\|_{L^{\infty}} \leq c\|f\|_{\dot{B}_{p, 1}^{r}} .
$$

2) If $f \in B_{p, 1}^{r}\left(\mathbb{R}^{d}\right)$ with $r \geq \frac{d}{p}$, then

$$
\|f\|_{L^{\infty}} \leq c\|f\|_{B_{p, 1}^{r}} .
$$


3) If $f \in B_{p, q}^{r}\left(\mathbb{R}^{d}\right)$ with $q>1$ and $r>\frac{d}{p}$, then

$$
\|f\|_{L^{\infty}} \leq c\|f\|_{B_{\infty, \infty}^{0}}\left(1+\log _{2} \frac{\|f\|_{B_{p, q}^{r}}}{\|f\|_{B_{\infty, \infty}^{0}}}\right) .
$$

In particular, (A.3) implies $\|f\|_{L^{\infty}} \leq c\|f\|_{B_{p, q}^{r}}$.

Proof. For $j \in \mathbb{Z},(2.8)$ allows us to write

$$
\Delta_{j} f=\sum_{|k-j|<2} \Delta_{k} \Delta_{j} f .
$$

It then follows from Proposition 2.2 that for any $1 \leq p \leq \infty$,

$$
\left\|\Delta_{j} f\right\|_{L^{\infty}} \leq c \sum_{|k-j|<2} 2^{\frac{k d}{p}}\left\|\Delta_{j} f\right\|_{L^{p}} \leq c 2^{\frac{j d}{p}}\left\|\Delta_{j} f\right\|_{L^{p}} .
$$

To prove (A.1), we assume $f \in \dot{B}_{p, 1}^{r}$ with $r=\frac{d}{p}$ and take the $L^{\infty}$-norm of $f=$ $\sum_{j=-\infty}^{\infty} \Delta_{j} f$ to get

$$
\|f\|_{L^{\infty}} \leq \sum_{j=-\infty}^{\infty}\left\|\Delta_{j} f\right\|_{L^{\infty}} \leq c \sum_{j=-\infty}^{\infty} 2^{\frac{j d}{p}}\left\|\Delta_{j} f\right\|_{L^{p}}=c\|f\|_{B_{p, 1}^{\frac{d}{p}}} .
$$

The proof of (A.2) is similar, but the difference is that $\Delta_{j}=0$ with $j \leq-2$ is zero in the context of a inhomogeneous Besov space. For $f \in B_{p, 1}^{r}$ with $r \geq d / p$,

$$
\begin{aligned}
\|f\|_{L^{\infty}} & \leq \sum_{j=-1}^{\infty}\left\|\Delta_{j} f\right\|_{L^{\infty}} \leq c \sum_{j=-1}^{\infty} 2^{\frac{j d}{p}}\left\|\Delta_{j} f\right\|_{L^{p}} \\
& \leq c \sum_{j=-1}^{\infty} 2^{j r}\left\|\Delta_{j} f\right\|_{L^{p}}=c\|f\|_{B_{p, 1}^{r}} .
\end{aligned}
$$

To prove (A.3), we write for $f \in B_{p, q}^{r}$

$$
f=\sum_{j=-1}^{\infty} \Delta_{j} f=\sum_{j=-1}^{N} \Delta_{j} f+\sum_{j=N+1}^{\infty} \Delta_{j} f,
$$

where $N$ is an integer to be specified. The $L^{\infty}$-norm of the first sum can be bounded by

$$
\sum_{j=-1}^{N}\left\|\Delta_{j} f\right\|_{L^{\infty}} \leq(N+2)\|f\|_{B_{\infty, \infty}^{0}} .
$$

while the second sum is bounded by

$$
\begin{aligned}
\sum_{j=N+1}^{\infty}\left\|\Delta_{j} f\right\|_{L^{\infty}} & \leq c \sum_{j=N+1}^{\infty} 2^{\frac{j d}{p}}\left\|\Delta_{j} f\right\|_{L^{p}} \\
& \leq c\left(\sum_{j=N+1}^{\infty} 2^{j q^{\prime}(d / p-r)}\right)^{1 / q^{\prime}}\left(\sum_{j=N+1}^{\infty} 2^{j r q}\left\|\Delta_{j} f\right\|_{L^{p}}^{q}\right)^{1 / q} \\
& \leq c 2^{-N q^{\prime}(r-d / p)}\|f\|_{B_{p, q}^{r},}
\end{aligned}
$$

where $q^{\prime}$ satisfies $1 / q+1 / q^{\prime}=1$. Thus, for a constant $c$ depending on $p, q$ and $r$,

$$
\|f\|_{L^{\infty}} \leq(N+2)\|f\|_{B_{\infty, \infty}^{0}}+c 2^{-N q^{\prime}(r-d / p)}\|f\|_{B_{p, q}^{r}} .
$$


If we set $N=O\left(\frac{1}{r-d / p} \log _{2} \frac{\|f\|_{B_{p, q}^{r}}}{\|f\|_{B_{\infty}^{0}, \infty}}\right),(\mathrm{A} .3)$ is then established.

We have used extensively the commutator estimates stated in the next two propositions. These estimates have previously been obtained in [16].

Proposition A.2. For $p \in[1, \infty]$ and $j \in \mathbb{Z}$, we have

$$
\left\|\left[u \cdot \nabla, \Delta_{j}\right] v\right\|_{L^{p}} \leq c\left(\|\nabla u\|_{L^{\infty}}\left\|\Delta_{j} v\right\|_{L^{p}}+\|\nabla v\|_{L^{\infty}}\left\|\Delta_{j} u\right\|_{L^{p}}\right),
$$

where the brackets [,] represent the commutator, namely

$$
\left[u \cdot \nabla, \Delta_{j}\right] v=u \cdot \nabla \Delta_{j} v-\Delta_{j}(u \cdot \nabla v) .
$$

The estimate in Proposition A.2 is suitable for situations when $u$ and $v$ are equally regular. If $\nabla v$ is not known to be in $L^{\infty}$, then the following commutator estimator is more useful.

Proposition A.3. For $p \in[1, \infty]$ and $j \in \mathbb{Z}$, we have

$$
\left\|\left[u \cdot \nabla, \Delta_{j}\right] v\right\|_{L^{p}} \leq c\left(\|\nabla u\|_{L^{\infty}}\left\|\Delta_{j} v\right\|_{L^{p}}+2^{j}\|v\|_{L^{\infty}}\left\|\Delta_{j} u\right\|_{L^{p}}\right)
$$

The following proposition bounds the product $u v$ in a Besov space in terms of the norms of $u$ and $v$ in the same Besov space.

Proposition A.4. For any $s>0$ and $p, q \in[1, \infty]$, we have

$$
\begin{aligned}
& \|u v\|_{\dot{B}_{p, q}^{s}} \leq c\left(\|u\|_{L^{\infty}}\|v\|_{\dot{B}_{p, q}^{s}}+\|v\|_{L^{\infty}}\|u\|_{\dot{B}_{p, q}^{s}}\right), \\
& \|u v\|_{B_{p, q}^{s}} \leq c\left(\|u\|_{L^{\infty}}\|v\|_{B_{p, q}^{s}}+\|v\|_{L^{\infty}}\|u\|_{B_{p, q}^{s}}\right) .
\end{aligned}
$$

The proof of this proposition is classical (see e.g. $[\mathbf{4}],[\mathbf{6}]$ ). A special consequence of this proposition and Proposition A.1 is that $\dot{B}_{p, 1}^{s}$ with $s=d / p, B_{p, 1}^{s}$ with $p \geq d / p$ and $B_{p, q}^{s}$ with $s>d / p$ are all Banach algebras.

\section{Acknowledgments}

I thank my colleague David Ullrich for discussions on the Littlewood-Paley theory, and the referee for pointing out the results of Cannone, Planchon and Lemarié-Rieusset.

\section{References}

[1] H. Bahouri and J.-Y. Chemin, Equations de transport relatives à des champs de vecteurs non-lipschitziens et mécanique des fluids, Arch. Rat. Mech. Anal. 127 (1994), 159-199.

[2] J. Bergh and J. Löfström, Interpolation Spaces, An Introduction, Springer-Verlag, BerlinHeidelberg-New York, 1976.

[3] M. Cannone, Ondelettes, Paraproduits et Navier-Stokes, Diderot Éditeur, Paris, 1995.

[4] D. Chae, Local existence and blow-up criterion for the Euler equations in the Besov spaces, RIMGARC preprint no. 01-7.

[5] D. Chae and J. Lee, Global well-posedness in the super-critical dissipative quasi-geostrophic equations, Commun. Math. Phys. 233 (2003), 297-311.

[6] J.-Y. Chemin, Perfect Incompressible Fluids, Clarendon Press, Oxford, 1998.

[7] R. Danchin, Local theory in critical spaces for compressible viscous and heat-conductive gases, Commun. Partial Differential Equations 26 (2001), 1183-1233. 
[8] P.G. Lemarié-Rieusset, Recent Developments in the Navier-Stokes Problem, Chapman \& Hall/CRC Research Notes in Mathematics 431, Chapman \& Hall/CRC, Boca Raton, FL, 2002.

[9] J. Peetre, New Thoughts on Besov Spaces, Duke University Press, 1976.

[10] E. Stein, Singular Integrals and Differentiability Properties of Functions, Princeton University Press, 1970.

[11] D. Ullrich, private communications.

[12] M. Vishik, Hydrodynamics in Besov spaces, Arch. Rat. Mech. Anal. 145 (1998), 197-214.

[13] M. Vishik, Incompressible flows of an ideal fluid with vorticity in borderline spaces of Besov type, Ann. Sci. École Norm. Sup. 32 (1999), 769-812.

[14] J. Wu, Generalized MHD equations, J. Differential Equations 195 (2003), 284-312.

[15] J. Wu, Global solutions of the 2D dissipative quasi-geostrophic equation in Besov spaces, SIAM J. Math. Anal., in press.

[16] J. Wu, Solutions of the 2D quasi-geostrophic equation in Hölder spaces, submitted.

Department of Mathematics, Oklahoma State University, Stillwater, OK74078, USA

E-mail address: jiahong@math.okstate.edu 